\title{
Corrigendum: In-channel integration of designable microoptical devices using flat scaffold-supported femtosecond-laser microfabrication for coupling-free optofluidic cell counting
}

Dong Wu, Jian Xu, Li-Gang Niu, Si-Zhu Wu, Katsumi Midorikawa and Koji Sugioka

Light: Science \& Applications (2015) 4, e275; doi:10.1038/Isa.2015.48; published online 13 February 2015

Correction to: Light: Science \& Applications (2015) 4, e228; doi:10.1038/lsa.2015.1; published online 2 January 2015

In this paper, in the "Materials and methods" section, the type of laser from IMRA America, Inc. was wrongly given as Er-fiber. The correct type is $\mathrm{Yb}$-doped fiber.

We apologize for any inconvenience this may have caused. 